\title{
VIES INÉGALES, MORTS INÉGALES
}

Analyse du profil des victimes d'homicide dans la ville de São Paulo*

Quelle analyse socio-économique de la violence au Brésil peut-on présenter, à partir des dernières données disponibles, sur le profil des victimes d'homicides dans la ville de São Paulo, réputée la plus violente de ce pays? Il faut prendre en compte le sexe, l'âge, l'ethnie, la profession et la zone de résidence de la victime, ainsi que l'évolution dynamique de cette violence, comparée avec des indicateurs révélateurs de la décadence économique de la ville, tels le chômage et le développement du trafic des drogues. Cela permet alors d'avancer quelques hypothèses concernant l'origine et l'évolution d'une telle violence.

Pour qu'un tel niveau de violence en termes d'homicides ait lieu, il faut que les ressources économiques et les biens publics soient abondants mais très inégalement répartis. Plus qu'un simple problème économique, la violence reflète donc un problème de légitimation, lorsque le régime de répartition de richesses est remis en cause par les couches sociales marginalisées de la société - d'où la nécessité de soulever les aspects sociaux, historiques et politiques de ce phénomène.

Vida desigual, morte desigual

\section{Análise do perfil das vítimas de homicídio na cidade de São Paulo}

Qual análise sócio-econômica da violência no Brasil se pode apresentar a partir dos últimos dados disponíveis sobre o perfil de vítimas de homicídios na cidade de São Paulo, considerada a mais violenta do país? A análise descritiva leva em conta o sexo, a idade, a raça, a profissão e a zona de residência da vítima, bem como a evolução dinâmica dessa violência, comparada com indicadores associados à decadência econômica da cidade, tais como o desemprego e o desenvolvimento do tráfico de drogas. Isso permitiu esboçar algumas hipóteses com relação à origem e à evolução de tal violência.

Para que um tal nível de violência em termos de homicídios ocorra, é necessário que os recursos econômicos e os bens públicos sejam abundantes mas extremamente mal distribuídos. Mais do que apenas um problema econômico, a violência remete a um problema de legitimação, quando o regime de repartição de riquezas é questionado pelas camadas marginalizadas da sociedade - o que explica a necessidade de salientar os aspectos sociais, históricos e políticos desse fenômeno.

\section{Unequal life, Unequal Death \\ Analysis of the Profile of Homicide Victims in São Paulo}

This paper presents a socioeconomic analysis of violence in Brazil, using the latest available data concerning the profile of homicide victims in São Paulo, known as the most violent city in this country. The variables considered by the descriptive analysis are sex, age, race, profession and the neighborhood of the victims. This data made possible an analysis of the dynamic evolution of this violence,

* Je tiens à remercier Pierre Salama, Blandine Destremeau, Jean-François Deluchey, MarieAgnès Lafage, Maryse Nunes et Elyamine Soum pour leurs commentaires. 
in relation with indicators of the city's economical decadence such as unemployment and drug traffic.

The descriptive analysis led to the formulation of some hypotheses about the origin and the evolution of this violence. For such a level of violence to exist, the economic resources and public goods must be abundant but very unequally distributed. More than a mere economic problem, violence reflects a question of legitimization - hence the need to highlight its social, historical and political aspects.

\author{
Comme tu te sens bien \\ au fond de ta tombe \\ C'est la part qui te revient \\ des biens de ce monde \\ Ce n'est pas un palais \\ ce n'est qu'une tombe \\ Mais pour le mort que tu es \\ elle est assez profonde 1
}

$\mathrm{L}$ 'actuel sentiment d'insécurité au Brésil ne s'explique pas seulement par une augmentation du niveau de la violence ces dernières années. C'est la nature même de cette violence qui a changé. Depuis les années quatre-vingt-dix, on observe le transfert d'une violence criminelle auparavant focalisée sur le patrimoine, vers des crimes contre la personne. La logique qui est derrière ces deux formes de violence n'est pas du tout la même. Si, pour les crimes contre le patrimoine, les individus les plus aisés de la société sont des cibles privilégiées, en revanche, en ce qui concerne les crimes contre les personnes, ce sont les plus pauvres qui sont le plus souvent les victimes de morts violentes. Dans cette analyse, on mettra l'accent sur la violence en termes d'homicides, sans pour autant nier l'importance des autres types de violence ou des corrélations entre les diverses formes sous lesquelles peut se présenter le crime dans les grandes villes brésiliennes. Simplement, la complexité du phénomène exige une analyse ciblée et description détaillée de son fonctionnement. Quels rapports existent-ils entre, d'une part, les transformations de la dynamique et de la structure de la violence, et, d'autre part, les transformations économiques et sociales subies par la ville de São Paulo ces dernières années? Cette question sera le fil conducteur de l'analyse. Il convient alors de décrire de façon détaillée le phénomène des homicides au vu des statistiques disponibles, de relever les régions où se manifeste cette violence, ainsi que le profil des victimes et l'évolution historique de cette violence.

En 2003, plus de 5000 homicides ont été dénombrés pour la seule ville de São Paulo, ce qui la place parmi les plus violentes du monde. Ce nombre montrerait déjà la gravité du problème, mais la violence est une réalité beaucoup plus complexe et d'autres facteurs contribuent à renforcer l'actuel sentiment d'insécurité. Les crimes contre la propriété ont été en nette progression ces dernières années - voitures et banques étant les principales cibles. De même, les crimes

\footnotetext{
${ }^{1}$ J. Cabral de Mello Neto, Mort et vie séverine (extraits), 1956. Édition en portugais chez Nova Fronteira, São Paulo, 2000.
} 
contre les personnes ont non seulement cru, mais se sont de plus en plus manifestés par des enlèvements. Cependant, le crime contre la vie, sous la forme de l'homicide, du vol suivi de meurtres ou tueries, constitue l'aspect le plus dramatique de la violence présente dans la ville de São Paulo. Tel sera ici l'objet principal.

Beaucoup d'études ont démontré l'importance du rapport entre l'évolution du nombre de victimes d'homicides et le contexte économique. Mais, derrière l'aggravation de la violence se cache un vaste univers. Ainsi, le profil des victimes d'homicides peut permettre de mieux comprendre l'influence des paramètres socioéconomiques sur la violence, et d'avancer quelques hypothèses sur son origine. Il faut évaluer la répartition de la violence dans la ville en fonction des classes sociales, des zones d'habitation, des ethnies, groupes professionnels, tranches d'âge et genres. Premièrement, il faudra analyser l'impact des inégalités dans la répartition du revenu et dans l'accès aux biens publics, sur la violence en termes d'homicides. Deuxièmement, il faut tenter de savoir pourquoi les pauvres sont les principales victimes. Enfin, l'aspect dynamique de la violence doit être évalué dans la ville en général et dans des zones spécifiques.

\section{Profil des victimes d'homicides à São Paulo}

Le choix de la ville de São Paulo est fondé à la fois sur la disponibilité des données mais aussi sur sa représentativité économique, sociale et démographique au sein de la région latino-américaine. De plus, les homicides sont devenus la principale cause de mortalité depuis les années 1980, devant les maladies cardiaques. Pour les jeunes de 15 à 24 ans, ce type de mort violente représente déjà $63 \%$ du total général des décès. Pour les seules cinq dernières années, on compte un total de 28000 homicides. Ce phénomène semble aller de pair avec la propagation du trafic de drogue et le processus de délocalisation industrielle vers d'autres régions du Brésil et l'étranger. De façon plus générale, on ne peut s'empêcher de l'associer à la réduction du rôle de l'État, notamment en matière d'éducation et de sécurité, et aux conséquences de la privatisation des entreprises publiques.

Analyser où, comment, par qui et pourquoi ont été tuées ces personnes permet d'aller au-delà d'une simple évaluation du niveau de la violence, puisque cela informe sur les conditions dans lesquelles vivaient les victimes et sur celles de leur mort. Cet objectif semble, a priori, plutôt descriptif, mais il s'avère important pour l'interprétation du phénomène. Par ailleurs, une évaluation des aspects analytiques du profil des victimes est d'autant plus pertinente qu'elle n'a pas encore fait suffisamment l'objet d'une analyse systématique de la part des chercheurs en sciences sociales au Brésil ${ }^{2}$.

Comment la structure des inégalités explique-t-elle la reproduction de la violence? Certaines contradictions inhérentes à la structure de répartition des richesses (et,

\footnotetext{
${ }^{2}$ Parmi les trop rares incursions sur le sujet, on peut citer: P. Borlina MaiA, "Vinte anos de homicídios no estado de São Paulo ", São Paulo em Perspectiva (São Paulo), XIII (4), 2000 : 121-129; N. CARdia, «Exposição à violência: seus efeitos sobre valores e crenças em relação a violência, polícia e direitos humanos », in C. Goirand (ed.), «Violence et contrôle de la violence au Brésil, en Afrique et à Goa », Lusotopie 2003 (Paris, Karthala), 2004 : 299-328.
} 
par voie de conséquence, des possibilités d'y accéder) dans la ville sont évidemment des mécanismes importants qui la nourrissent: le racisme, le trafic de drogue et le crime organisé (sous la forme de braquages, enlèvements, vols dans des institutions financières et commerciales), la ségrégation résidentielle et la polarisation centre-périphérie (en termes économiques, politiques et sociaux). Ces aspects sont, à la fois, en forte contradiction avec le procès d'accumulation - puisque de nature excluante - et avec les valeurs et principes de la modernisation, censés être universalistes et démocratiques ${ }^{3}$. Bref, en dévoilant la structure socio-économique de la violence par le biais de l'analyse du profil des victimes d'homicides, on doit pouvoir progresser dans la compréhension de son maintien et de sa reproduction dans un pays sous-développé comme le Brésil.

São Paulo, avec une population d'un peu plus de dix millions d'habitants, est la plus grande ville brésilienne, et une des plus grandes du monde. Fondée en 1554 , la ville restera très peu développée et peuplée jusqu'à la fin du XIX ${ }^{\mathrm{e}}$ siècle. Son essor économique s'explique par deux faits marquants dans l'histoire du pays. Le premier est le succès de l'entreprise caféière dans la zone sud-orientale du Brésil à partir de 1880, dont la ville de São Paulo bénéficiera en tant qu'important centre financier et de distribution des marchandises. Le second est le début du processus d'industrialisation que connaîtra São Paulo et son plus grand développement à partir des années 1930. Ces deux événements historiques sont aussi liés au mouvement migratoire vers la ville, attirant à la fois les étrangers, venus surtout d'Europe, mais aussi des migrants de l'intérieur originaires des zones rurales peu développées. São Paulo atteint ainsi son premier million d'habitants dans les années 1930, et, suite à plusieurs vagues de migration, la ville a vu le nombre de sa population multiplié par huit entre 1940 et $1980^{4}$.

\section{Polarisation centre-périphérie}

Capitale de la région la plus industrialisée du pays, São Paulo subit néanmoins, à l'heure actuelle, une grave crise économique, à la fois conjoncturelle et structurelle. Conjoncturelle, car l'activité économique, fondée sur le secteur des services, décroît, en conséquence de la stagnation du taux de croissance au Brésil depuis deux décennies. Structurelle, car la région dans laquelle se trouve la ville connaît une longue période de transformation de son activité productive, dont l'aspect le plus marquant est la déconcentration industrielle vers d'autres régions plus attractives en termes de coûts de production ${ }^{5}$. La spécialisation actuelle concerne les services financiers et le commerce. Néanmoins, la région métropolitaine, dans

\footnotetext{
${ }^{3}$ Pour une discussion approfondie du développement contradictoire du système capitaliste par rapport à sa base idéologique, voir I. Walllerstein, Le capitalisme historique, Paris, La Découverte, 1996 : 79-84.

${ }^{4}$ Selon la Fundação Sistema Estadual de Análise de Dados (SEADE).

5 Pour une description détaillée de ce processus, voir B. NEGRI, Concentração e desconcentração industrial em São Paulo (1880-1990), Campinas, Editora da Unicamp, 1996 ; une analyse plus récente peut être trouvée chez E. Marques \& H. Torres, «São Paulo no contexto do sistema mundial de cidades », Novos Estudos, São Paulo, Cebrap, 56, 2000.
} 
laquelle se trouve la ville, totalise toujours près d'un quart de la production industrielle du pays, et est le siège des plus grandes entreprises multinationales en activité au Brésil 6 .

La délocalisation des industries, les transformations dans la composition organique du capital (dues notamment à la révolution informatique) et la monopolisation de la production ont pour conséquence une diminution drastique du nombre d'emplois disponibles. À São Paulo, le taux de chômage atteint actuellement environs 20 \% de la population active - ce qui est deux fois plus élevé que le niveau moyen observé dans les années 1980. L'économie informelle est, par conséquent, devenue la principale source de revenu pour la majorité de la population. Le nombre de personnes travaillant au noir a doublé ces dix dernières années ${ }^{7}$.

Ces transformations économiques dans le monde du travail ont eu de fortes répercussions sur le niveau de pauvreté dans la ville. Malgré les effets, au départ positifs, du Plan Real en termes de répartition de richesses, la pauvreté a continué de croître dans les années $1990^{8}$. Le revenu moyen des travailleurs de la région métropolitaine de São Paulo a diminué de $20 \%$ lors des dix dernières années 9 . Les non-Blancs, plus particulièrement touchés par la pauvreté et l'indigence dans la ville, occupent la majorité des emplois dits précaires. Ils constituent la majorité du nombre de chômeurs, des personnes sans domicile fixe et des habitants de bidonvilles ${ }^{10}$.

La capitale de l'État, en plus de ces caractéristiques économiques, présente des particularismes urbains. Pour rendre plus accessible son administration, elle a été divisée en sept zones regroupant 96 quartiers (ou districts). Le critère utilisé pour ce regroupement a été fondamentalement géographique, n’obéissant donc pas aux différences socio-économiques existant entre les divers quartiers de la ville. Pourtant, la logique d'occupation urbaine garde une forte correspondance avec celle de la répartition des richesses. Ainsi, la proximité avec le centre-ville a permis l'accès à la propriété d'immeubles commerciaux et résidentiels dans ces zones bénéficiant des infrastructures économiques et du réseau des transports. Les quartiers les plus éloignés ont été occupés plus récemment et manquent d'infrastructures et d'accès aux services publics.

Dès lors, les quartiers des zones Sud-Ouest et Centre concentrent la population la plus riche de la ville. Les classes moyennes résident les quartiers situés dans les zones Nord et Nord-Ouest, alors que la population pauvre se regroupe dans les zones Sud, Sud-Est et Est. Il convient pourtant de nuancer ce constat.

${ }^{6}$ E. Marques \& H. Torres, «São Paulo no contexto . . . », op. cit.

7 Voir B. Lautier, L'économie informelle dans le tiers monde, Paris, La Découverte, 1994.

${ }^{8}$ Selon Sónia Rocha, la proportion de pauvres vivant au-dessous du seuil de pauvreté se situerait aux alentours de $25 \%$ de la population, d'après les critères de la Banque mondiale, «Pobreza e desigualdade no Brasil : o esgotamento dos efeitos distributivos do Plano Real », Rio de Janeiro-Brasilia, Ministério Do Planejamento, Orçamento e Gestão, Instituto de Pesquisa Econômica Aplicada, 2000 («Texto para discussão », 721).

${ }^{9}$ Pesquisa de emprego e desemprego (PED), IBGE, 2000.

${ }^{10}$ Pour une analyse détaillée des statistiques concernant les populations des bidonvilles et sans domicile fixe, consulter les recensements réalisés par la FIPE (Fundação Instituto de Pesquisas Econômicas), de l'Université de São Paulo, respectivement en 1995 et 2000. 
D'une part, la zone centrale subit un processus de détérioration depuis au moins deux décennies, entraînant la chute de la valeur des immeubles et, par conséquent, la venue d'une population issue de couches sociales défavorisées, voire misérables (occupant certains bâtiments sous la forme de squats). D'autre part, quelques « îlots de prospérité » subsistent dans certaines zones plus éloignées du centre. C'est ainsi le cas des zones Sud, Nord et Nord-Ouest. Toutefois, il ne s'agit là que d'exceptions qui n'affecteront pas beaucoup le sens de notre analyse.

Le revenu moyen des habitants est très inégalement réparti entre les diverses zones de la ville. Selon ce critère, mesurant la pauvreté monétaire, la zone la plus pauvre est la zone Est de la ville qui, avec $23 \%$ de la population, ne rassemble que $4 \%$ des ménages à haut revenu. Les zones les plus éloignées du centre-villeSud, Sud-Est et Est - concentrent un peu moins de $70 \%$ des ménages à bas revenu. En revanche, la zone Sud-Ouest, la plus riche, ne représente que $12 \%$ de la population et accueille $43 \%$ des ménages à haut revenu. Le tableau I classe, par ordre décroissant, les zones concentrant les ménages à haut revenu dans le total de ménages. Ce classement sera utilisé tout au long de notre analyse.

La ville de São Paulo présente ainsi un cadre de forte polarisation de type centre-périphérie pour ce qui concerne le revenu de ses habitants. Mais, ce n'est pas seulement le revenu qui y est mal réparti. Les équipements et services publics - écoles, crèches, hôpitaux - sont de très mauvaise qualité dans les zones les plus éloignées, qui sont par ailleurs très mal desservies par le réseau de transports urbains. Le tableau II présente quelques variables socio-économiques qui caractérisent la qualité de vie des habitants de cette ville. Pour que l'analyse ne reste pas excessivement centrée sur l'aspect monétaire de la vie de ces personnes, nous avons choisi aussi d'autres variables concernant leurs niveaux de santé et d'éducation.

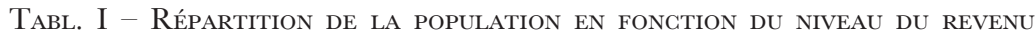
DES MÉNAges PAR zONES À SÃo PaUlo EN 2000

\begin{tabular}{lrrrrrrr}
\hline Zone & $\begin{array}{c}\text { Population } \\
\text { totale }\end{array}$ & $\begin{array}{c}\% \text { du } \\
\text { total }\end{array}$ & $\begin{array}{c}\text { Ménages à bas } \\
\text { revenu** }\end{array}$ & $\begin{array}{c}\% \text { du } \\
\text { total }\end{array}$ & $\begin{array}{c}\text { Ménages à haut } \\
\text { revenu } \text { *** }^{*}\end{array}$ & $\begin{array}{c}\% d u \\
\text { total }\end{array}$ & $\begin{array}{c}\% \text { dans la } \\
\text { zone }\end{array}$ \\
\hline Sud-Ouest & 1243927 & 11,9 & 39040 & 6,5 & 120487 & 42,7 & 9,7 \\
Centre & 413896 & 4,0 & 15959 & 2,7 & 27226 & 9,7 & 6,6 \\
Nord-Ouest & 1244598 & 11,9 & 70834 & 11,8 & 32373 & 11,5 & 2,6 \\
Nord & 1118418 & 10,7 & 60225 & 10,1 & 25134 & 8,9 & 2,2 \\
Sud-Est & 1869646 & 17,9 & 105524 & 17,6 & 37061 & 13,1 & 2,0 \\
Sud & 2188806 & 21,0 & 149313 & 25,0 & 28048 & 9,9 & 1,3 \\
Est & 2354961 & 22,6 & 157199 & 26,3 & 11658 & 4,1 & 0,5 \\
Total & 10434252 & 100,0 & 598094 & 100,0 & 281987 & 100,0 & 2,7 \\
\hline
\end{tabular}

Source: Recensement national, IBGE.

* Estimation 2002.

** Revenu du chef de famille compris entre 1,5 et 3,0 salaires minimum, en 2000.

*** Revenu du chef de famille au-dessus de 20 salaires minimum, en 2000. 
Tabl. II - Répartition RELATive DE LA POPUlation PAR zONE EN FONGTion DE L'incidence de la pauvreté, de la mortalité infantile, des Personnes ÂGÉEs, DE l'analphabétisme et de leur niveau universitaire - SÃo Paulo, 2000

\begin{tabular}{|c|c|c|c|c|c|c|}
\hline Zone & $\begin{array}{c}\text { Population } \\
\text { totale }\end{array}$ & $\begin{array}{c}\text { Population } \\
\text { pauvre* }^{*}\end{array}$ & $\begin{array}{l}\text { Mortalité } \\
\text { infantile** }\end{array}$ & $\begin{array}{l}\text { Personnes } \\
\text { âgées*** }\end{array}$ & Analphabétisme ***** $^{*}$ & $\begin{array}{c}\text { Niveau } \\
\text { universitaire }^{* * * *}\end{array}$ \\
\hline Sud-Ouest & $11,9 \%$ & $6,5 \%$ & $7,0 \%$ & $19,9 \%$ & $6,3 \%$ & $36,6 \%$ \\
\hline Centre & $4,0 \%$ & $2,7 \%$ & $3,3 \%$ & $7,8 \%$ & $1,4 \%$ & $11,3 \%$ \\
\hline Nord-Ouest & $11,9 \%$ & $11,8 \%$ & $12,5 \%$ & $12,2 \%$ & $11,3 \%$ & $11,5 \%$ \\
\hline Nord & $10,7 \%$ & $10,1 \%$ & $9,7 \%$ & $13,1 \%$ & $9,3 \%$ & $9,7 \%$ \\
\hline Sud-Est & $17,9 \%$ & $17,6 \%$ & $14,8 \%$ & $21,3 \%$ & $16,4 \%$ & $13,7 \%$ \\
\hline Sud & $21,0 \%$ & $25,0 \%$ & $27,3 \%$ & $10,9 \%$ & $29,5 \%$ & $10,4 \%$ \\
\hline Est & $22,6 \%$ & $26,3 \%$ & $25,4 \%$ & $14,9 \%$ & $25,9 \%$ & $6,8 \%$ \\
\hline Total & $100,0 \%$ & $100,0 \%$ & $100,0 \%$ & $100,0 \%$ & $100,0 \%$ & $100,0 \%$ \\
\hline
\end{tabular}

Source: Recensement national, IBGE, 2000.

* Pourcentage de ménages à bas revenu (voir tableau I).

** Enfants de 0 à 4 ans.

*** Au-dessus de 70 ans.

**** Situation du chef de famille.

\section{Dynamique et structure de la violence à São Paulo}

Quel est le profil des victimes d'homicide dans la ville de São Paulo? Dans l'analyse, on cherchera tout d'abord à dresser une évaluation de la structure actuelle du profil des victimes, à travers les caractéristiques ressortant de la base de données que sont le genre, la race, l'âge, la situation professionnelle, sera nécessaire. Puis, on cherchera à présenter la dimension dynamique de cette violence, c'est-à-dire les transformations de niveau sur une plus longue période.

La mise en évidence de ces aspects structurels dévoile une face cachée de la violence au Brésil. Cela ne veut pas dire que les analyses qui se penchent sur l'étendue du phénomène - son évolution dynamique - n'ont pas d'importance. Simplement, plonger à l'intérieur de cette violence peut renseigner davantage sur l'essence de son fonctionnement.

La violence, en termes d'homicides dans la ville de São Paulo, s'est sensiblement accrue pendant les années quatre-vingt-dix. Certes, au cours de la dernière année, le nombre d'homicides a chuté de 7,7\%. Cette décrue résulte des équipements de sécurité, de la mise en place de politiques sociales luttant contre la pauvreté, du déplacement de la violence vers d'autres villes de la région et du programme national de suppression des armes à feu. Néanmoins, cette diminution récente des homicides n'obère pas la tendance sur le long terme. La progression par rapport à 1993 a été de $31 \%$ et de $190 \%$ par rapport à 1983, ce qui correspond à une croissance annuelle de l'ordre de $5 \%$ ces vingt dernières années.

Certes, la délinquance sous forme de crimes contre le patrimoine et contre la personne augmente, elle aussi, très rapidement. Mais l'importance prise par 


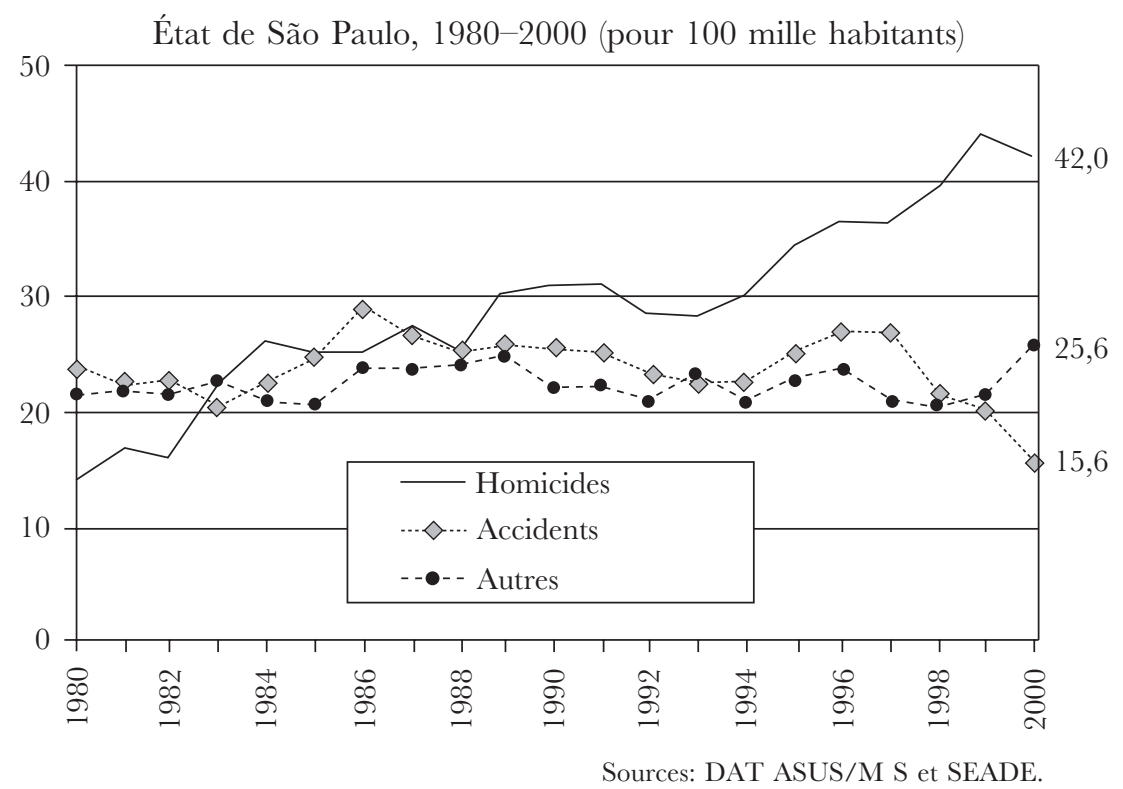

Graphique 1 : Taux de mortalité par cause externe

l'homicide ces derniers temps est l'aspect le plus frappant de cette violence. Comme on peut le voir à travers le graphique 1, à partir de la fin des années quatrevingt, les homicides sont la première cause externe (violente) de mort dans l'État de São Paulo, dépassant les accidents de la route et toutes les autres formes de décès (parmi lesquelles, le suicide). Si en 1980 les homicides représentaient à peine $20 \%$ de la mortalité par cause externe, aujourd'hui ils en représentent un peu moins de la moitié.

Pourtant, ni les homicides, ni leur évolution, ne sont uniformément répartis entre les divers districts et zones qui composent la ville de São Paulo. En 2003, le district le plus violent fut le Grajaú (zone Sud), qui a enregistré 327 homicides. En revanche, Moema (zone Sud-Ouest), l'un des quartiers les plus aisés de la ville, n'en recense qu'un. Le tableau suivant rassemble ces informations en fonction de la zone de résidence de la victime.

La réduction de la violence ces cinq dernières années est due en grande partie à la variation observée dans la zone Sud-Ouest, la plus aisée de la ville. On remarque par ailleurs que la violence se combine avec la détérioration des conditions d'habitation. Durant les dix dernières années, dans les quartiers de banlieue, la croissance démographique a parfois dépassé la barre des $30 \%$. Dans ces quartiers périphériques, les nouveaux arrivants s'installent dans des bidonvilles et doivent se battre pour la possession du terrain ainsi que pour l'accès à l'eau et à l'électricité. Cette instabilité, démographique et économique, se manifeste notamment par une lutte pour des ressources essentielles et explique en bonne partie, outre l'intervention des trafiquants, l'essor de la violence. En revanche, dans des quartiers situés au centre, le niveau de victimes est très bas par rapport à la périphérie. Ainsi, les zones Nord-Ouest et Sud-Ouest enregistrent une baisse considérable sur la période, 
Tabl. III - RÉPARTition du NOMBRE DE VICTIMES D'Homicide Par zone D'habitation À São Paulo

\begin{tabular}{lcrrrr}
\hline Zone & $\begin{array}{c}\text { Homicides } 2003 \\
(A)\end{array}$ & $\%$ & $\begin{array}{r}\text { Homicides } 1998 \\
(B)\end{array}$ & $\%$ & Évolution $(A) /(B)$ \\
\hline Sud-Ouest & 249 & $5,0 \%$ & 348 & $6,9 \%$ & $-28,4 \%$ \\
Centre & 127 & $2,5 \%$ & 116 & $2,2 \%$ & $9,5 \%$ \\
Nord-Ouest & 501 & $10,0 \%$ & 515 & $9,8 \%$ & $-2,7 \%$ \\
Nord & 405 & $8,1 \%$ & 361 & $6,9 \%$ & $12,2 \%$ \\
Sud-Est & 726 & $14,5 \%$ & 810 & $15,4 \%$ & $-10,4 \%$ \\
Sud & 1514 & $30,2 \%$ & 1317 & $25,1 \%$ & $15,0 \%$ \\
Est & 114 & $22,2 \%$ & 1095 & $20,8 \%$ & $1,7 \%$ \\
Ignorée & 379 & $7,6 \%$ & 695 & $13,2 \%$ & $-45,5 \%$ \\
Total & 5015 & $100,0 \%$ & 5257 & $104,8 \%$ & $-4,6 \%$ \\
\hline
\end{tabular}

Source: Pro-Aim (Programa de Aprimoramento de Informações sobre Mortalidade), Mairie de São Paulo.

ce qui reflète la montée en puissance des dépenses en faveur des équipements de sécurité publique et surtout privée (gardiens armés, véhicules blindés et sécurisation technologique des maisons).

De plus, il faut souligner que la répartition des homicides ne correspond pas à celle de la population dans la ville. Dans certaines zones le pourcentage d'homicides est relativement élevé compte tenu de la population qui y réside. Ainsi, la zone Sud-Ouest compte $5 \%$ du total des victimes en 2003, alors que sa population représente $12 \%$ de la population totale de São Paulo. En revanche, la zone Sud est marquée par un plus grand contraste: $30 \%$ des victimes d'homicides pour $21 \%$ de la population.

La façon la plus objective d'observer ces répartitions consiste à calculer le taux d'homicides en fonction de la population. À São Paulo, on dénombre 48 homicides pour 100000 habitants, alors que ce chiffre était seulement de 14 en 1982, soit une augmentation de $300 \%$, et une progression de $7 \%$ par an. Parmi les trente quartiers qui enregistrent les taux d'homicides les plus élevés, deux seulement se trouvent dans une zone centrale ${ }^{11}$, tous les autres sont situés en banlieue. C'est ce que nous montre le tableau IV. L'une des zones les plus peuplées et pauvres, celle du Sud, détient le plus grand taux d'homicides. La probabilité qu'un habitant de cette zone y soit assassiné est 3,3 fois plus élevée que dans la zone Sud-Ouest, la plus riche. Le taux d'homicides dans cette zone est comparable à certains pays en situation de guerre civile. A contrario, la probabilité d'homicides pour les habitants des quartiers les plus riches est comparable à celle du Danemark.

${ }^{11}$ Il s'agit du Brás et de la Sé, quartiers qui ont connu un très fort développement du trafic de « crack» ces dernières années. 
Tabl. IV. - Zone D'habitation et TAuX D'Homicides POUR ioo ooo habitants - São Paulo, 2003

\begin{tabular}{|c|c|c|}
\hline Zone & $\begin{array}{c}\text { Homicides } 2003 \\
\text { nombre absolu de victimes }\end{array}$ & $\begin{array}{l}\text { Taux d'homicides } 2003 \\
\text { nombre de victimes pour } 100000 \text { hab. }\end{array}$ \\
\hline Sud-Ouest & 249 & 20,4 \\
\hline Centre & 127 & 32,2 \\
\hline Nord-Ouest & 501 & 39,4 \\
\hline Nord & 405 & 36,5 \\
\hline Sud-Est & 726 & 38,8 \\
\hline Sud & 1514 & 66,5 \\
\hline Est & 1114 & 46,1 \\
\hline Total & 5015 & 47,5 \\
\hline
\end{tabular}

Source: Pro-Aim, Mairie de São Paulo et IBGE.

Le graphique 2 illustre cette comparaison entre la probabilité d'homicide et le revenu des habitants de la ville. On observe ainsi une relation négative, comme on pouvait s'y attendre, entre les taux d'homicide et le niveau de revenu des habitants. Néanmoins, quoique la relation soit forte, le revenu n'explique qu'un peu plus de la moitié de la localisation des homicides dans la ville. C'est pourquoi nous ne pouvons pas nous limiter à ce seul élément. Il faut nécessairement en chercher d'autres pour comprendre ce phénomène.

Le chômage est une autre variable économique à laquelle la violence est intimement liée, comme le montre le graphique 3. Sur une longue période, la crise

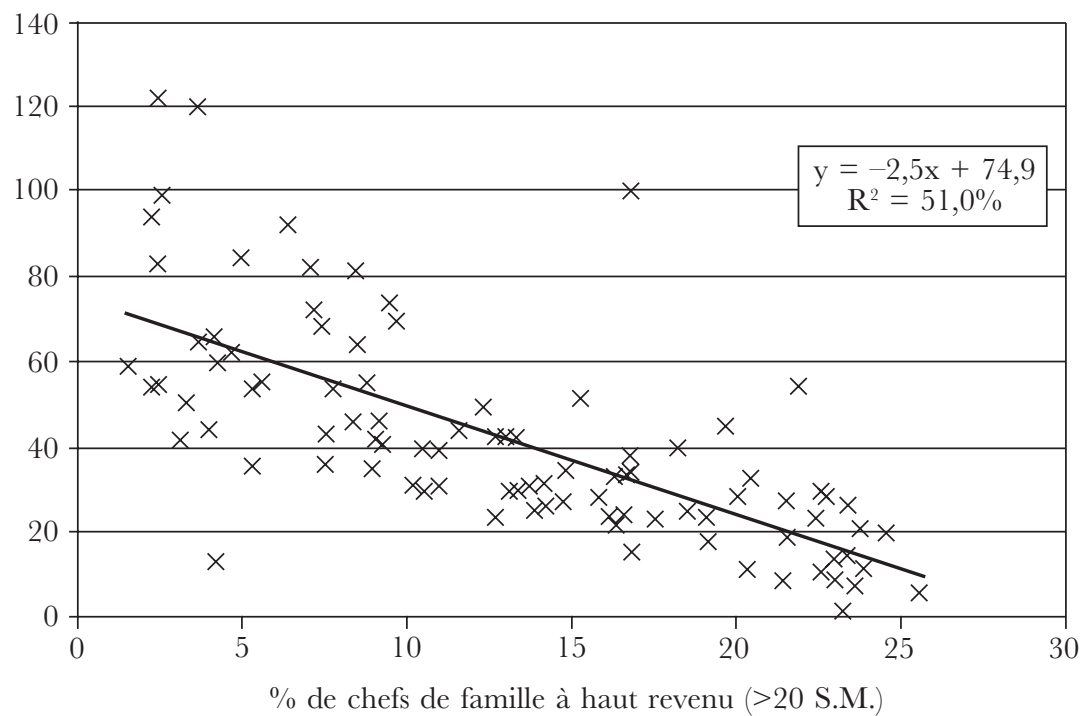

Graphique 2 : Répartition du revenu et des taux d'homicides par district de résidence de la victime São Paulownt2000 from Brill.comø4/26/2023 03:33:07PM 


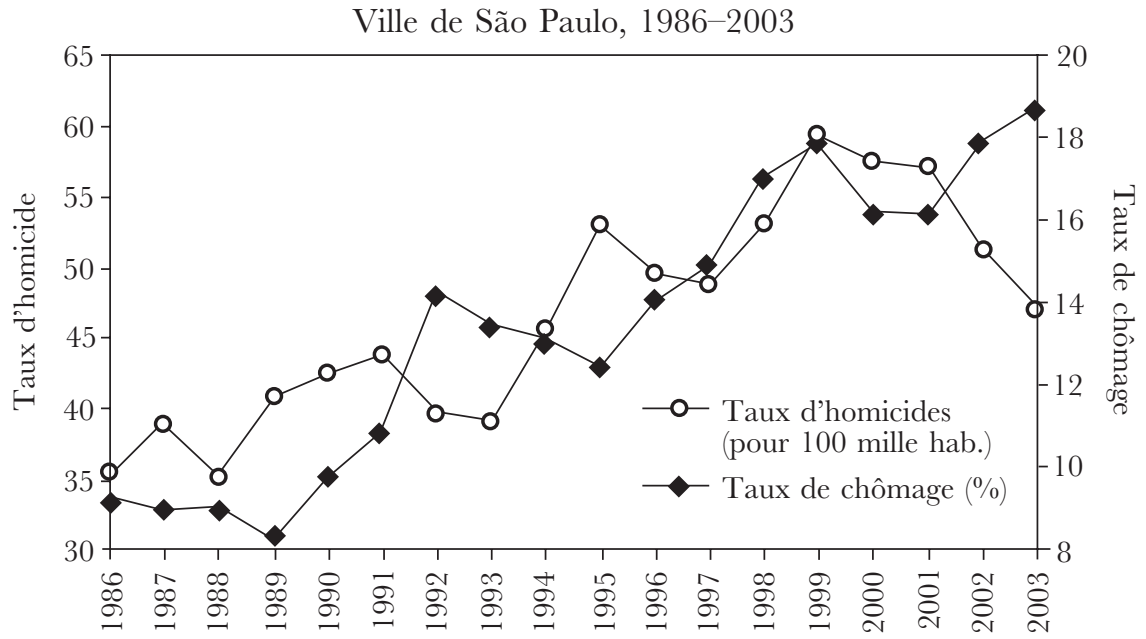

Graphique 3 : Évolution des taux d'homicides et de chômage

économique que subit la ville de São Paulo, dont l'aspect le plus marqué est la diminution des postes formels dans le marché du travail, se répercute plus visiblement sur le taux d'homicides. Le crime devient une activité alternative pour certains groupes sociaux.

Les travailleurs non qualifiés (conducteurs de véhicules, maçons, vendeurs, gardiens d'immeubles) prédominent parmi les victimes d'homicides à São Paulo. Le graphique 4 le prouve de façon très claire. Dans une position sociale marginalisée, ils sont les plus susceptibles d'être directement ou indirectement mêlés au monde du crime. La criminalité - «activité » certes très risquée, mais rentable attire de plus en plus ces groupes défavorisés qui recherchent des petits boulots dans l'économie de la drogue ou qui commettent des crimes divers pour obtenir une source alternative de revenus.

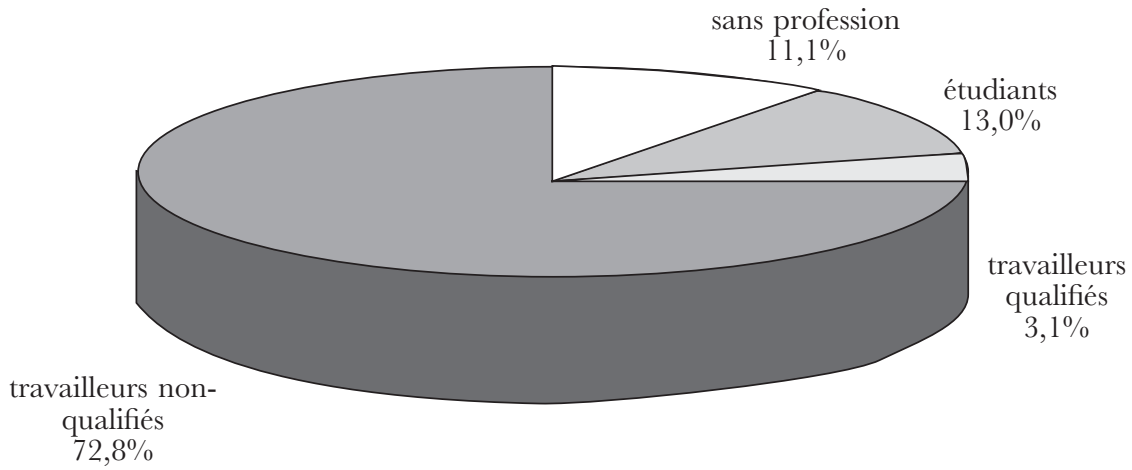

Source: Pro-Aim, Mairie de São Paulo

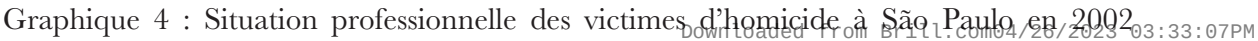


En 2002, les homicides à São Paulo visaient, pour une large majorité, les jeunes et les adolescents. Ces deux groupes rassemblent presque $60 \%$ du nombre total de victimes comme l'indique le tableau $\mathrm{V}$. Le chômage est un phénomène beaucoup plus présent dans cette tranche de la population qui subit également une pression de plus en plus forte de la société de consommation. Ce sont là deux facteurs qui incitent fortement les jeunes à participer au monde du crime et pourraient expliquer leur plus grande vulnérabilité à la violence.

Tabl. V - RÉPARTition des Victimes D'HOMicides Par tranches D’Âge São Paulo, 2003

\begin{tabular}{lccc}
\hline Groupe & Age compris entre & Nombre de victimes & $\%$ \\
\hline Enfants & 0 et 14 ans & 72 & $1,4 \%$ \\
Adolescents & 15 et 19 ans & 837 & $16,7 \%$ \\
Jeunes & 20 et 29 ans & 2127 & $42,4 \%$ \\
Adultes & 30 et 59 ans & 1768 & $35,3 \%$ \\
Personnes âgées & plus de 59 ans & 86 & $1,7 \%$ \\
Ignoré & - & 125 & $2,5 \%$ \\
Total & - & 5015 & $100,0 \%$ \\
\hline
\end{tabular}

Source: Pro-Aim, Mairie de São Paulo, 2003.

En ce qui concerne le sexe des victimes, on observe une très forte prédominance des hommes (93\% en 2003). La violence contre les femmes est, toutefois, en progression ces dernières années (4,5\% sur sept années, face à 3,2 \% chez les hommes). Plus de 2400 assassinats de femmes à São Paulo depuis 1997 attestent de l'importance du problème. Par ailleurs, c'est une violence qui semble avoir une logique un peu différente de celle exercée contre les hommes, puisque l'univers domestique y joue un rôle plus important ${ }^{12}$. Ainsi, en 1999, la moitié des homicides de jeunes filles ont été commis dans leurs propres maisons, contre seulement $17 \%$ chez les jeunes garçons ${ }^{13}$.

Un facteur fondamental pour comprendre le niveau de violence à São Paulo est sans doute la couleur de la peau des victimes d'homicide. Le fait d'être noir ou métis semble augmenter la probabilité de devenir une cible de la violence dans la ville. Ainsi, si les non-Blancs ne représentent qu'une minorité de $32 \%$ dans la population, ils constituaient en 2003 la moitié des victimes d'homicide, ce qui révèle une forte surreprésentation, selon le tableau VI.

${ }^{12}$ À propos de l'importance de la sphère domestique dans la violence sexuelle au Brésil, voir A.L. Paixão \& G.C. Filho Beato, «Crimes, vítimas e policiais », Tempo Social (São Paulo, USP), IX (1), 1997.

${ }^{13}$ La participation des femmes dans la violence ne se limite pourtant pas à un rôle passif, tel qu'on l'a montré ici. Une analyse très riche de la participation active des femmes aux homicides au Brésil a été faite par R. de Oliveira Almeida, Mulheres que matam, Rio de Janeiro, Relume Dumará, 2001. 
Tabl. VI - RÉPARTition des Victimes D'Homicide PAR RAGE/COUleur DE PEAU SÃo Paulo, 2003

\begin{tabular}{lcc}
\hline Groupe & Population totale & Victimes d'homicides \\
\hline Blancs & $67,8 \%$ & $49,8 \%$ \\
Non-Blancs* & $32,2 \%$ & $50,2 \%$ \\
Total & $100,0 \%$ & $100,0 \%$ \\
\hline
\end{tabular}

Sources: Pro-Aim, Mairie de São Paulo, 2003 et IBGE, 2000.

* Correspond au regroupement de Noirs et métis.

Cette surreprésentation des non-Blancs dans les statistiques de la violence à São Paulo s'explique en partie par leur forte présence dans les quartiers les plus pauvres de la ville, là où justement la violence se manifeste de façon plus forte ${ }^{14}$. Cependant, cela n'est pas suffisant pour expliquer la prédominance des Noirs et des métis parmi les victimes d'homicide, puisque même dans certaines zones à majorité blanche, les homicides des non-Blancs restent importants. Il en va ainsi par exemple de la zone Sud-Ouest, la plus riche de la ville, où une minorité inférieure à $10 \%$ de non-Blancs dans la population résidente a représenté en 2003, $43 \%$ des victimes ${ }^{15}$.

On peut émettre quelques hypothèses sur l'identité des agresseurs en se penchant sur le profil des victimes. Premièrement, une piste très importante est la zone de résidence dont sont issues ces dernières. Bien évidemment, dans la majorité des cas, il s'agit de régions où le trafic de drogue est très présent. D’ailleurs, une enquête menée par la police civile de l'État de São Paulo indiquait que le motif principal de plus d'un tiers des tueries était lié au trafic de drogue ${ }^{16}$. Deuxièmement, on remarque que la quasi-totalité des meurtres ont été commis avec une arme à feu, ce qui implique que les agresseurs y ont facilement accès. Troisièmement, la violence policière est très présente dans la région. Ainsi, selon le rapport de la police civile cité auparavant ${ }^{17}$, sur vingt homicides de jeunes en 1999, un était commis par un policier.

${ }^{14}$ Cela rejoint les résultats de l'enquête menée par N. CARDIA, qui montrent la plus grande vulnérabilité des populations noires et métisses de la banlieue de la ville à ce type de violence («Exposição à violência ....", op. cit.).

15 L'association race et violence a été l'objet de nombreuses études, dont notamment: J. Gorender, Brasil em Preto e Branco, São Paulo, Senac, 2000 ; J. da Silva, « Representação e ação dos operadores do sistema penal no Rio de Janeiro », Tempo Social, IX (1), 1997 ; J. Domingues VARgas, «Indivíduos sob suspeita : A cor dos acusados de estupro no fluxo do sistema de justiça criminal », Dados (Rio de Janeiro, IUPERJ), XLII (4), 1999 ; C.A. Costa RibeIro, Cor e Criminalidade. Estudo e análise da justiça no Rio de faneiro (1900-1930), Rio de Janeiro, UFRJ, 1995.

${ }^{16}$ Cependant, la majorité des agresseurs n'a pas pu être identifiée avec exactitude (Ouvidoria da Polícia do Estado de São Paulo, Relatório Anual de Prestação de Contas, São Paulo, Imprensa Oficial,1999).

17 Entre 1981 et 1991, la police avait été jugée coupable de l'assassinat de 5535 personnes à São Paulo. Néanmoins, de nombreux meurtres sont probablement masqués par la police et 
Puisque le trafic de drogue constitue un élément fondamental dans l'essor de la violence à São Paulo, il est important d'y consacrer une attention soutenue. Dans les années 1970, le Brésil est devenu un haut lieu de passage de la cocaïne reliant les pays producteurs (Colombie, Bolivie et Pérou) à l'Europe et aux ÉtatsUnis. Un réseau de transports ferroviaires, routiers, maritimes et aériens fit de l'État de São Paulo une région potentielle pour l'écoulement de la drogue. La montée en puissance de ce réseau pendant les années 1980, associée notamment à la recrudescence des conflits dans les pays producteurs, a marqué le changement du rôle du pays dans le système mondial de la drogue, d'un simple espace de transit à une importante aire de consommation, voire de production de drogue (plus développée pour la marijuana que pour la cocaïne, du moins jusqu'à l'heure actuelle). Toutefois le Brésil reste une région plutôt intermédiaire dans ce système, orienté vers les marchés nord-américains et européens, où se trouve la majorité des consommateurs et où se concentrent les principaux bénéficiaires de ce commerce. Au Brésil, l'économie de la drogue emploie un million de personnes, dans des fonctions diverses en petits groupes dispersés dans toutes les régions du pays. À São Paulo, ceux qui en tirent le plus grand profit ne sont pourtant pas les petits trafiquants (qui constituent la majorité de la population carcérale et qui s'exposent davantage à la violence), mais les intermédiaires responsables de la logistique et du transport de la drogue. Il s'agit, en général, de grands commerçants et de sociétés privées, de propriétaires de terres ou d'avions et d'entreprises de transport routier.

Le développement du trafic de drogue entraîne l'augmentation de la violence au Brésil. Mais on ne pourra pas déterminer précisément qui sont les auteurs des homicides à São Paulo, puisque, entre autres problèmes, beaucoup de procès n'aboutissent pas à une condamnation ${ }^{18}$. Toutefois, sur la base de travaux divers, on peut avancer quelques explications pour rendre compte du phénomène de la violence tel qu'il se manifeste à São Paulo actuellement.

* * *

Les caractéristiques dominantes qui ressortent de l'analyse de l'univers des victimes d'homicide sont: noires, de sexe masculin, jeunes, habitant la banlieue et très peu qualifiées. Le profil des victimes d'homicide coïncide donc de très près avec celui de la population pauvre de la ville de São Paulo. La pauvreté y est noire, selon des lignes de ségrégation géographiques, touchant essentiellement les plus jeunes et les moins adaptés aux besoins du marché du travail. La thèse du rapprochement entre la pauvreté et la violence doit être donc analysée de près. Elle est l'objet de nombreuses controverses dans la littérature consacrée au sujet. Il ne s'agit évidemment pas de défendre la thèse d'un déterminisme économique

enregistrés comme «résultats de conflits armés ». Voir à ce propos : A.L. Paixão \& G.C. Beato F., «Crimes ... », op. cit., où les auteurs cherchent, entre autre, à expliquer la peur de la police par les habitants des grandes villes brésiliennes.

${ }^{18}$ Sur les inégalités devant la justice criminelle au Brésil, voir l'analyse de S. Adorno, «Violence urbaine, justice criminelle et organisation sociale du crime », Cahiers du Brésil contemporain (Paris, EHESS), 31, 1997. 
pur et dur de la violence. Il vaut la peine, par conséquent, de rappeler à grands traits ce débat, pour proposer ensuite une approche inclusive des multiples contours du phénomène. S'il est vrai que la pauvreté dans une société donnée amène la violence, ou du moins un certain degré de violence, comme l'avait suggéré Karl Marx ${ }^{19}$, on devrait alors observer des conflits peu ou prou identiques un peu partout dans le monde: la misère est loin d'être un «privilège » brésilien. Or, Haïti, pour prendre un exemple extrême et rester dans la même région, est un État qui ne connaît pas, sur le moyen terme, le même niveau d'homicides que le Brésil $^{20}$. De plus, il n'y a pas que les pauvres qui sont assassinés. Ils forment certes l'immense majorité des victimes, mais la violence contre les classes aisées et moyennes a pris de l'importance ces dernières années, ce qui reflète une contestation de l'ordre économique et du régime de répartition des richesses. Le développement de cette violence s'explique ainsi par une logique différente de celle qui touche les couches les plus pauvres. Ici, on a affaire à une utilisation de la force essentiellement pour extorquer de l'argent par vols à main armée, enlèvements de courte ou longue durée et crimes contre la propriété suivie de meurtres.

La violence déborde, en effet. Mais les quartiers les plus riches restent toujours beaucoup mieux «sécurisés » que ceux de la périphérie. La prédominance des pauvres dans les statistiques d'homicides est ainsi le résultat indirect de la façon dont s'organise cette société. La polarisation de la richesse dans la ville rend plus facile le développement du trafic de drogue qui trouve parmi les plus aisés une demande importante et parmi les plus misérables une main-d'œuvre bon marché. Les rivalités internes de pouvoir augmentent à leur tour la violence en terme d'homicides dans des groupes déjà défavorisés.

Deux observations empiriques s'imposent. En premier lieu, un exemple régional. Le déplacement de l'activité économique de São Paulo (et des mécanismes inhérents qui reproduisent les inégalités) vers d'autres régions de l'État entraîne, par voie de conséquence, un déplacement de la violence ${ }^{21}$. En second lieu, d'autres grands centres urbains et économiques dans le monde, tels que Washington et Johannesbourg, ont connu des périodes de très grande criminalité. Ainsi, là où se trouvent les richesses économiques importantes et là où ces richesses sont très inégalement réparties (tout comme l'ensemble des biens publics et des opportunités en général), on peut observer des contestations et des conflits, puisque les richesses seront perçues comme illégitimes. Ceci constitue un aspect fondamental ${ }^{22}$. Autrement dit,

19 «Dès qu'ils (les jeunes) deviennent trop âgés pour leur travail d'enfant, donc au plus tard à l'âge de 17 ans, on les renvoie de l'imprimerie. Ils sont alors candidats à la criminalité. Diverses tentatives pour leur procurer un emploi ailleurs ont échoué du fait de leur ignorance, de leur grossièreté et de leur délabrement physique et intellectuel », K. Marx, Le Capital. Livre I, Paris, Quadrige - PUF, 1993 : 545. (1 $1^{\mathrm{e}}$ éd. : 1872)

${ }^{20}$ A. Peralva ( Democracia e violência: a modernização por baixo », in "As transições e a modernidade ", Lua Nova. Revista de cultura e política (São Paulo, Centro de Estudos de Cultura Contemporânea), 40-41, 199) remarque aussi que même pour le cas brésilien, la carte de la misère ne correspond pas à la carte de la violence.

${ }^{21}$ Ainsi, ces dernières années, Campinas (à $100 \mathrm{~km}$ de la capitale) et Ribeirão Preto (à $300 \mathrm{~km}$ ) sont devenues des villes très riches, inégales et violentes.

${ }^{22}$ Il faut, bien évidemment, faire la différence avec le phénomène de la violence d'origine politique visant la conquête du pouvoir d'une nation ou d'un territoire (Colombie, Sri Lanka). 
pour qu'il y ait autant de morts, pour que la ville soit comparable à un champ de bataille ou à une région en guerre civile, il faut qu'il y ait un problème majeur de légitimité de l'ordre politique et économique dans la société. Ce n'est donc pas tellement le crime organisé, dont le trafic est l'une des facettes, qui est la raison ultime des homicides. C'est plutôt lorsqu'il est désorganisé que cela se produit ${ }^{23}$. La violence est ainsi un moyen, et non une fin en soi : celui d'obtenir du pouvoir, des richesses et d'exercer une domination sur un groupe de personnes.

Il faut aussi analyser les autres éléments, non économiques, pouvant expliquer ce phénomène: il s'agit de la dimension historique, de la propagation du trafic d'armes et de drogue, des questions de genre et de race, et enfin du rôle de l'État. Le caractère violent fondateur de la nation brésilienne est perçu à travers trois événements majeurs de l'histoire: la «découverte» du Brésil par les colons portugais et le massacre des peuples indigènes qui s'ensuivit; les trois siècles d'esclavage ${ }^{24}$; les vingt ans de répression sous le régime militaire (1964-1985) ${ }^{25}$. La voie - prédominamment et non pas ponctuellement - conflictuelle de cette formation sociale dans l'histoire est en contradiction directe avec l'apparente et prétendue cordialité de ses rapports sociaux.

L'économie inégale qui s'enracine dans les grands centres urbains du Brésil, associée au trafic de drogue et d'armes, est un élément central de compréhension de la violence. La combinaison de ces deux facteurs est problématique. En effet, on l'a vu, quasiment toutes les victimes d'homicide ont été tuées par une arme à feu et, si l'on ne peut y discerner directement une influence du trafic de drogue, l'origine géographique des victimes coïncide pour le moins avec les régions de manifestation et de contrôle de ce trafic. Pourtant, il serait trop simple d'imputer aux «pauvres» l'essor de cette économie inégale et de la violence. Le trafic de drogue ne subsisterait pas s'il n'y avait pas demande dans les quartiers aisés de la ville. Bref, il n'existe pas de pauvreté sans que des riches n'en tirent profit. Et, il n'existe pas non plus de richesse sans qu'il n'y ait des pauvres qui soient là pour l'entretenir.

Aucun des éléments soulevés ci-dessus n'explique à lui seul la structure de la violence à São Paulo. Cependant, il n'est pas difficile de trouver chez certains auteurs ou certains courants de pensée une tendance à accentuer le déterminisme de l'un ou l'autre de ces éléments. Une attitude opposée, mais également regrettable, est l'insistance sur les spécificités régionales de la violence et le caractère prétendument apolitique de l'origine du phénomène, dénotant la vision relativiste d'une telle approche. Certes, la spécificité régionale, sociale et économique doit être prise en compte. Le phénomène de la violence en particulier à São Paulo, et, plus générale-

${ }^{23}$ "Quand la criminalité est efficacement "organisée", elle peut rendre moins visible le recours à la violence physique », C. Caldeira, «El crimen organizado en Brasil. Tendencias del control penal », Nueva Sociedad, 167, 2000 : 107, note 22.

${ }^{24}$ Pour certains auteurs, cela constituerait aussi un massacre, ou génocide, envers les populations noires. Voir A. do Nascimento, O genocídio do negro brasileiro. Processo de um racismo mascarado, Rio de Janeiro, Paz e Terra, 1978.

${ }^{25}$ Pour une analyse plus détaillée des racines historiques de la violence au Brésil, consulter J. Gorender, Brasil em Preto...., op. cit. et «História e Violência», Anais do VII Encontro Regional da Associą̧ão Nacional de História, Rio de Janeiro, UERJ, 1996. 
ment, au Brésil, ne se présente pas de la même façon qu'à Medellín, Johannesbourg ou Washington. La spécificité de São Paulo tenant à la répartition des revenus et des services publics, à sa constitution ethnique et à son histoire économique, a été soulignée ailleurs dans ce texte. Pourtant, on ne saurait ignorer le lien entre la centralisation extrême du pouvoir politique et économique dans cette ville et les manifestations de violence. La forme contradictoire selon laquelle se produisent et se reproduisent les richesses, entraînant une grave polarisation à la fois géographique, économique, sociale, culturelle et raciale dans la ville, est la clé des récentes évolutions dans la structure et la dynamique de la violence à São Paulo. La violence se présente donc comme l'expression ultime des contradictions spécifiques du processus d'accumulation du capital en cours au Brésil, le remettant en cause de la façon la plus extrême.

Université de Paris 3, Institut des hautes études de l'Amérique latine CAPES (Gouvernement du Brésil) [Révision du texte par Éric Morier-Genoud, Victor Pereira et Michel Cahen] 